\title{
Studies on the Interaction Parameter in Polysiloxane Solutions
}

\author{
Akihiko Muramoto \\ Material Research Laboratory, Toshiba Research and Development Center, \\ Kawasaki, Kanagawa, Japan.
}

(Received April 9, 1970)

\begin{abstract}
The thermodynamic properties of solutions of polydimethylsiloxane and silicon resin in non-polar solvents have been studied in a moderate concentration range at temperatures from 26 to $40^{\circ} \mathrm{C}$. Applying Flory's theory, the interaction parameter $\chi_{1}$ was calculated from the activity of solvent, which was determined from the lowering of its vapor pressure. It was found that the value of $\chi_{1}$ and its temperature dependency are characteristic of the interaction of polysiloxane with solvent. The heat term $\left(\Delta W_{h}\right)$ and the entropy term $\left(\Delta W_{s}\right)$ in $\chi_{1}$ were calculated from the temperature dependency of $\chi_{1}$ at the average temperature. It was confirmed that the obtained relation between $\Delta W_{h}$ and $\Delta W_{s}$ is represented by a smooth curve, independent of concentration and solvent which goes nearly through the origin.

KEY WORDS Polydimethylsiloxane / Silicone Resin / Thermodynamics / Interaction Parameter / Polymer Solution /
\end{abstract}

The Flory-Huggins equation (eq 1) has been widely used in interpreting the thermodynamic properties of polymer-liquid mixtures.

$$
\Delta \mu_{1}=R T\left[\ln \left(1-v_{2}\right)+(1-1 / x) v_{2}+\chi_{1} v_{2}{ }^{2}\right]
$$

where $\Delta \mu_{1}$ is the difference in the chemical potential between the solvent in a polymer solution and the pure solvent, $x$ is the ratio of the molecular volumes of polymer and solvent, $v_{2}$ is the volume fraction of polymer in the solution, and $\chi_{1}$ is defined as a free energy parameter to characterize a given polymer-solvent pair at a specified temperature. ${ }^{1}$

$\chi_{1}$ has been obtained by measuring the vapor pressure of solvent on polymer solution ${ }^{2,3}$ or the osmotic pressure of solution. ${ }^{4}$ The vapor pressure method is the most adequate to measure the $\chi_{1}$ in a moderately concentrated solution of either linear or branched polymer.

The natural rubber-benzene system has been studied first. The $\chi_{1}$ in the system is found to be independent of the concentration over a wide range. ${ }^{5}$ However, generally speaking, $\chi_{1}$ depends on the concentration. For example, it has been show $^{6}$ that the $\chi_{1}$ for polystyrene solution increased in methylethylketone, but decreased in toluene, with increasing polymer concentration.
The separate contributions of the heat component and the entropy component have been studied by many workers. ${ }^{7}$ Recently, C. Booth and coworkers ${ }^{8}$ obtained the values of $\Delta W_{h}$ and $\Delta W_{s}$ from the values of $\chi_{1}$ at two temperatures for natural rubber in acetone, methyl ethyl ketone and ethyl acetate, and showed these obtained values of $\Delta W_{h}$ and $\Delta W_{s}$ were positive and increased with increasing polymer concentration. Takenaka has shown that the relation between $\Delta W_{h}$ and $\Delta W_{s}$ in a nitrocellulose--acetone system gives a straight line which goes through the origin. ${ }^{9}$

In this report, the thermodynamic properties of a solution of polysiloxanes in various nonpolar solvents were investigated in terms of the interaction parameter $\chi_{1}$, and of the heat and entropy components in $\chi_{1}$.

\section{THEORETICAL}

As a general representation of the free energy of mixing $n_{1}$ solvent molecules with $n_{2}$ polymer molecules to form a solution containing a volume fraction $v_{2}$ of polymer, the following equation has been given

$$
\Delta F_{M}=k T\left[n_{1} \ln v_{1}+n_{2} \ln v_{2}+\chi_{1} n_{1} v_{2}\right]
$$

$\chi_{1}$ is a parameter which characterizes the free energy change in the standard state, $k T_{\chi_{1}} n_{1} v_{2}{ }^{1}$, 
which has been assigned to the process, in which pure polymer and pure solvent are converted to the contact state of the two components in the solution. ${ }^{10}$

The entropy change, $\Delta S_{M}$, and the heat of mixing, $\Delta H_{M}$, are given by

$$
\begin{aligned}
\Delta S_{M} & =-\left(\partial F_{M} / \partial T\right) p \\
& \left.=\Delta S_{M}{ }^{*}-k\left[\partial \chi_{1} T\right) / \partial T\right] n_{1} v_{2}
\end{aligned}
$$

and

$$
\begin{aligned}
\Delta H_{M} & =-T^{2}\left[\partial\left(\Delta F_{M} / T\right) / \partial T\right]_{p} \\
& =-k T^{2}\left(\partial \chi_{1} / \partial T\right) n_{1} v_{2}
\end{aligned}
$$

where $\Delta S_{M}{ }^{*}$ is the configurational entropy change of mixing in the ideal case, ${ }^{1}$ that is

$$
\Delta S_{M}{ }^{*}=-k\left(n_{1} \ln v_{1}+n_{2} \ln v_{2}\right)
$$

and the term $-k\left[\partial\left(\chi_{1} T\right) / \partial T\right] n_{1} v_{2}$ represents the standard state entropy change. ${ }^{11}$ Representing the heat term and the entropy term in $\chi_{1}$ with $\Delta W_{h}$ and $\Delta W_{s}$, respectively, the following equation is defined, on the assumption that $\Delta W_{h}$ and $\Delta W_{s}$ are independent of temperature. ${ }^{1}$

$$
R T \chi_{1}=\Delta W_{h}-T \Delta W_{s}
$$

In this report, the value of $\chi_{1}$ was calculated by using eq 1 from the activity of the solvent, which was obtained by measuring the vapor pressure $p$ of solvent over the polymer solution, relative to that $p_{0}$ over pure solvent, in equilibrium.

\section{EXPERIMENTAL}

\section{Material}

Polydimethylsiloxane (I) and silicone resin (II) were synthesized by the ordinary method and were treated to cut low molecular portions by precipitation in methyl alcohol from a tetrachloro methane solution. The above silicone resin was poly (dimethyldiphenylsiloxane) with branched chains, in which the ratio of the methyl group to the phenyl group was 1.74 . Their molecules were shown to be flexible and noncrystalline by literature already published. ${ }^{12}$ The molecular weight was found to be 88000 for sample I by the intrinsic viscosity method, ${ }^{13}$ and 4200 for sample II by ebullioscopy with toluene. Their densities, which were shown in Figure 1, were measured by means of a picnometer.

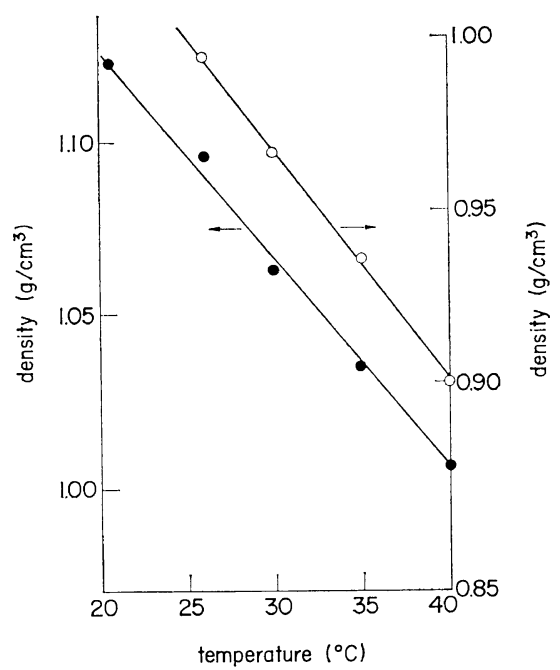

Figure 1. Density of polydimethylsiloxane (-O-) and silicone resin (- - )

Solvents used in this experiment were nonpolar solvents, that is, benzene, toluene, ethyl benzene, $n$-hexane, and $n$-octane. They were purified by distillation from commercial reagents and their purity was checked by gas chromatography. Their densities were referred from published data. ${ }^{14}$ Prepared solutions were left until mixing reached a uniform state. In particular, solutions in relatively higher concentrations were left for a few days to make sure they were completely uniform. Incomplete solutions could be checked by abnormal values of the ratio of $p / p_{0}$.

\section{Measurement of Vapor Pressure}

It was found, in the measurement of the ratio of $p / p_{0}$, that a large part of the error arose from the deviation of the temperature at which $p$ were measured from that at which $p_{0}$ were measured. So as to compensate for this, differential manometry was used according to the same principle described by Gee and Treloar. ${ }^{2}$ The manometer immersed in the constant temperature bath was controlled within an accuracy of $\pm 0.01^{\circ} \mathrm{C}$ and the head of mercury was read to $0.005 \mathrm{~cm}$ by a cathetometer. The relations of $p / p_{0} v s . v_{2}$ at measuring temperatures are shown in Figures 2 and 3 . 


\section{A. Muramoto}

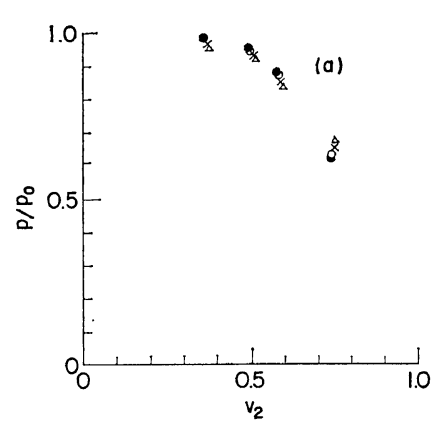

$$
v_{2}=\frac{1}{\frac{W_{1}}{W_{2}} \times \frac{\rho_{2}}{\rho_{1}}+1}
$$

where $\rho_{1}, \rho_{2}$ are the densities of solvent and solute, respectively.
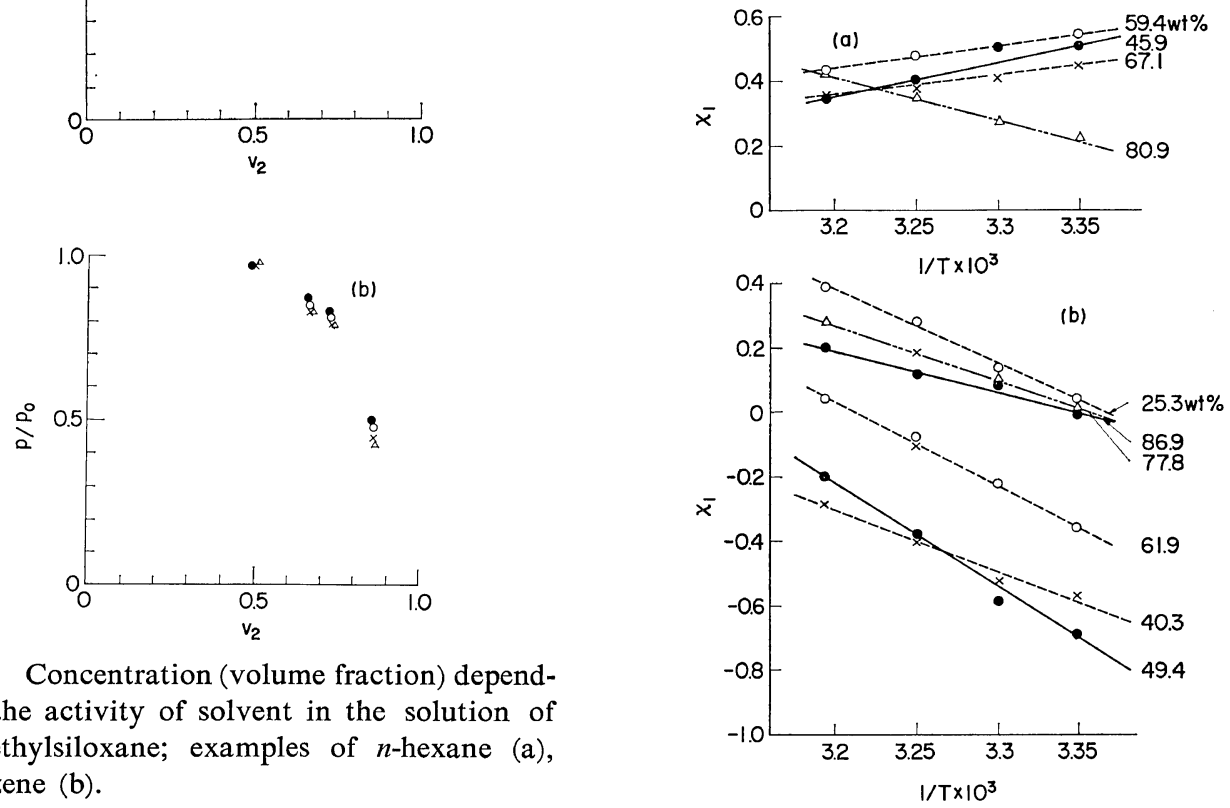

Figure 2. Concentration (volume fraction) dependence of the activity of solvent in the solution of polydimethylsiloxane; examples of $n$-hexane (a), and benzene (b).

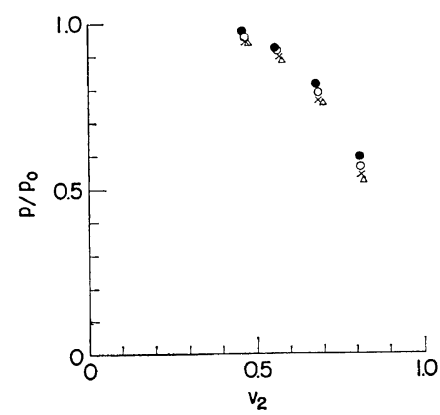

Figure 3. Concentration (volume fraction) dependence of the activity of solvent in the solution of silicone resin; an example of toluene.

\section{CALCULATION OF $\chi_{1}$}

The values of $\chi_{1}$ were calculated by eq 1 , where the term $1 / x$ was neglected as it was far less than unity. The values of $v_{2}$, assuming that no volume change occurred on mixing, were calculated at each temperature from the following equation
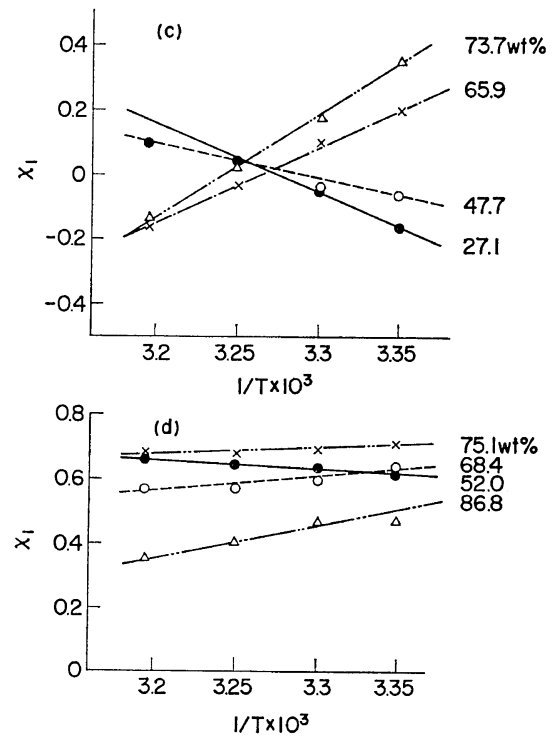

Figure 4. Temperature dependence of the interaction parameter $\chi_{1}$ for polydimethylsiloxane-solvent systems: (a) $n$-hexane, (b) $n$-heptane, (c) $n$-octane, (d) benzene, (e) toluene, and (f) ethylbenzene. 

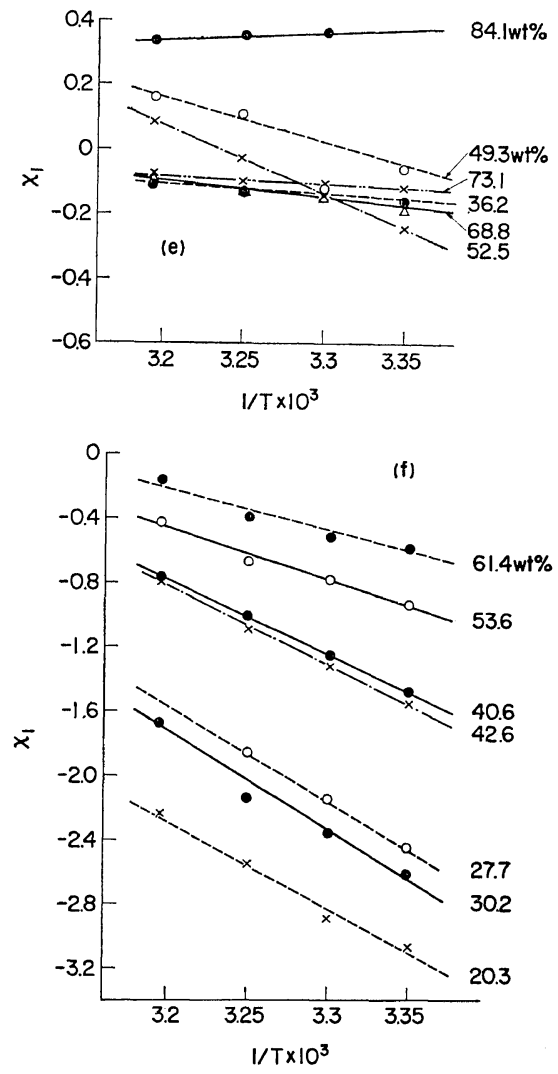

Figure 4. (continued)

On the assumption that the volume fraction of solute in a solution was constant in the range of temperature studied, the $\chi_{1}$ values were plotted against $1 / T$ in Figures 4 and 5 . The obtained graph was found to be a straight line relation. Then, using eq 6 , the resolution of $\chi_{1}$ into $\Delta W_{h}$ and $\Delta W_{s}$ terms was made from the slope and intercept, respectively. $\Delta W_{h}$ and $\Delta W_{s}$ obtained are listed in Tables I and II. Furthermore, $\Delta W_{h}$ was plotted against corresponding $\Delta W_{s}$ and a curve was obtained to show the relation between them.

\section{DISCUSSION}

The dependence of the activity of solvent on the polymer concentration has been estimated from the activity $v s$. volume fraction curves. ${ }^{15}$ The theoretical curve of the activity $v s$. volume fraction has been given by M. L. Huggins, ${ }^{15}$ on the assumption that the interaction parameter
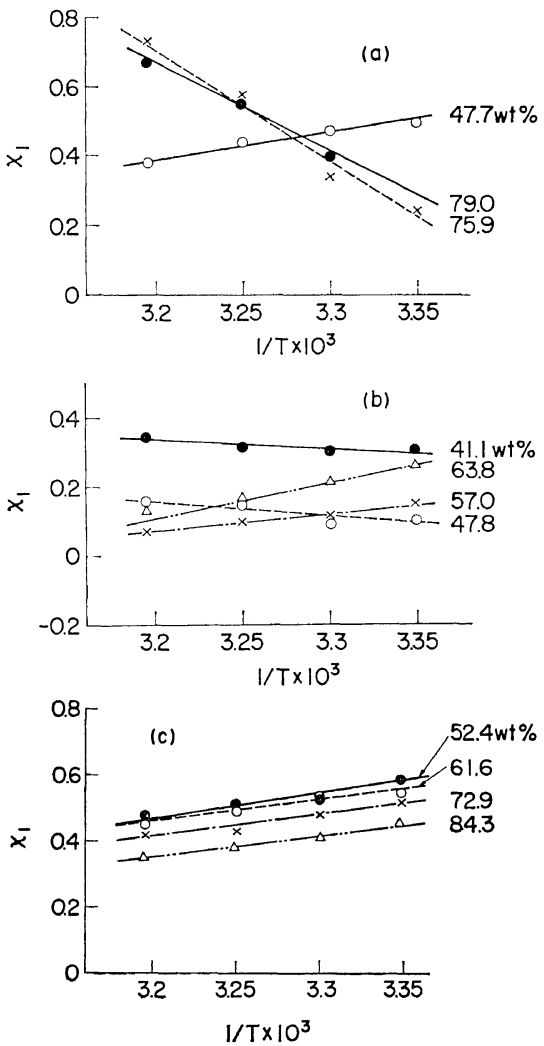

Figure 5. Temperature dependence of the interaction parameter $\chi_{1}$ for silicone resin-solvent systems: (a) n-octane, (b) benzene, and (c) toluene.

$\chi_{1}$ is independent of concentration at a constant temperature. An example of such a system is the solution of natural rubber in benzene investigated by G. Gee and L. R. G. Treloar. ${ }^{2}$ But such a solution as the interaction parameter being independent of concentration is rather fortuitous. For example, natural rubber in acetone, methyl ethyl ketone, or ethyl acetate, showed the concentration dependency in its interaction parameter. ${ }^{8}$ If the interaction parameter depends on concentration, the relation of the activity with concentration should deviate from the above theoretical relation curve.

In this experiment, the relations of the activity with concentration were shown in Figures 2 and 3. Since these relations showed that the activities of solvent decreased non-uniformly as the polymer concentration increased, the interaction parameter in the studied solution depends on the concentration. These figures show also 
Table I. $\Delta W_{h}$ and $\Delta W_{s}$ in polydimethylsiloxane solutions at $33^{\circ} \mathrm{C}$

\begin{tabular}{|c|c|c|c|c|c|c|c|c|c|c|c|c|c|c|}
\hline \multicolumn{5}{|c|}{ Solvent, $n$-hexane } & \multicolumn{5}{|c|}{ Solvent, $n$-heptane } & \multicolumn{5}{|c|}{ Solvent, $n$-octane } \\
\hline \multirow{2}{*}{$v_{2}$} & \multicolumn{2}{|c|}{$\chi_{1}$} & \multirow{2}{*}{$\Delta W_{h} \times 10^{-3}$} & \multirow{2}{*}{$\Delta W_{s}$} & \multirow{2}{*}{$v_{2}$} & \multicolumn{2}{|c|}{$\chi_{1}$} & \multirow{2}{*}{$\Delta W_{h} \times 10^{-3}$} & \multirow{2}{*}{$\Delta W_{s}$} & \multirow{2}{*}{$v_{2}$} & \multicolumn{2}{|c|}{$\chi_{1}$} & \multirow{2}{*}{$\Delta W_{h} \times 10^{-3}$} & \multirow{2}{*}{$\Delta W_{s}$} \\
\hline & $30^{\circ} \mathrm{C}$ & $40^{\circ} \mathrm{C}$ & & & & $30^{\circ} \mathrm{C}$ & $40^{\circ} \mathrm{C}$ & & & & $30^{\circ} \mathrm{C}$ & $40^{\circ} \mathrm{C}$ & & \\
\hline 0.367 & 0.46 & 0.35 & 2.1 & 6.3 & 0.194 & 0.15 & 0.39 & -4.5 & -15.8 & 0.215 & -0.05 & 0.17 & -4.2 & $-i 4.1$ \\
\hline 0.499 & 0.51 & 0.44 & 1.3 & 3.6 & 0.315 & -0.49 & -0.29 & -3.8 & -12.0 & 0.400 & -0.08 & 0.11 & -2.2 & -7.4 \\
\hline 0.582 & 0.42 & 0.36 & 1.2 & 3.1 & 0.413 & -0.54 & -0.20 & -6.4 & -20.7 & 0.585 & 0.09 & -0.36 & 8.4 & 28.4 \\
\hline \multirow[t]{3}{*}{0.746} & 0.28 & 0.42 & -2.6 & -9.5 & 0.535 & -0.23 & 0.05 & -5.3 & -17.5 & 0.672 & 0.19 & -0.14 & 6.3 & 21.1 \\
\hline & & & & & 0.712 & 0.10 & 0.28 & -3.4 & -11.8 & & & & & \\
\hline & & & & & 0.825 & 0.06 & 0.20 & -2.6 & -9.1 & & & & & \\
\hline
\end{tabular}

\begin{tabular}{|c|c|c|c|c|c|c|c|c|c|c|c|c|c|c|}
\hline \multicolumn{5}{|c|}{ Solvent, benzene } & \multicolumn{5}{|c|}{ Solvent, toluene } & \multicolumn{5}{|c|}{ Solvent, ethylbenzene } \\
\hline \multirow{2}{*}{$v_{2}$} & \multicolumn{2}{|c|}{$\chi_{1}$} & \multirow{2}{*}{$\Delta W_{h} \times 10^{-3}$} & \multirow{2}{*}{$\Delta W_{s}$} & \multirow{2}{*}{$v_{2}$} & \multicolumn{2}{|c|}{$\chi_{1}$} & \multirow{2}{*}{$\Delta W_{h} \times 10^{-3}$} & \multirow{2}{*}{$\Delta W_{s}$} & \multirow[b]{2}{*}{$v_{2}$} & \multicolumn{2}{|c|}{$\chi_{1}$} & \multirow{2}{*}{$\Delta W_{h} \times 10^{-3}$} & \multirow[b]{2}{*}{$\Delta W_{s}$} \\
\hline & $30^{\circ} \mathrm{C}$ & $40^{\circ} \mathrm{C}$ & & & & $30^{\circ} \mathrm{C}$ & $40^{\circ} \mathrm{C}$ & & & & $30^{\circ} \mathrm{C}$ & $40^{\circ} \mathrm{C}$ & & \\
\hline 0.496 & 0.62 & 0.66 & -0.74 & -3.7 & 0.338 & -0.14 & -0.11 & -0.58 & -1.7 & 0.187 & -2.83 & -2.26 & -10.8 & -41.3 \\
\hline 0.664 & 0.61 & 0.56 & -0.93 & -4.1 & 0.467 & 0.02 & 0.17 & -2.8 & -9.6 & 0.381 & -1.26 & -0.76 & -9.3 & -28.8 \\
\hline 0.731 & 0.70 & 0.68 & 0.40 & -0.036 & 0.499 & 0.14 & 0.09 & -4.3 & -14.4 & 0.401 & -1.31 & -0.80 & -9.8 & -30.6 \\
\hline \multirow[t]{3}{*}{0.857} & 0.46 & 0.35 & 2.0 & 6.0 & 0.664 & -0.15 & -0.09 & -1.1 & -3.4 & 0.510 & -0.79 & -0.45 & -6.5 & -20.3 \\
\hline & & & & & 0.700 & -0.11 & -0.09 & -0.42 & -1.2 & 0.589 & -0.48 & -0.21 & -4.8 & -15.5 \\
\hline & & & & & 0.827 & 0.36 & 0.34 & 0.42 & 0.70 & & & & & \\
\hline
\end{tabular}

Table II. $\Delta W_{h}$ and $\Delta W_{s}$ in silicone resin solutions at $33^{\circ} \mathrm{C}$

\begin{tabular}{|c|c|c|c|c|c|c|c|c|c|c|c|c|c|c|}
\hline \multicolumn{5}{|c|}{ Solvent, benzene } & \multicolumn{5}{|c|}{ Solvent, toluene } & \multicolumn{5}{|c|}{ Solvent, $n$-octane } \\
\hline \multirow{2}{*}{$v_{2}$} & \multicolumn{2}{|c|}{$\chi_{1}$} & \multirow{2}{*}{$\Delta W_{h} \times 10^{-3}$} & \multirow{2}{*}{$\Delta W_{s}$} & \multirow{2}{*}{$v_{2}$} & \multicolumn{2}{|c|}{$\chi_{1}$} & \multirow{2}{*}{$\Delta W_{h} \times 10^{-3}$} & \multirow{2}{*}{$\Delta W_{s}$} & \multirow{2}{*}{$v_{2}$} & \multicolumn{2}{|c|}{$\chi_{1}$} & \multirow{2}{*}{$\Delta W_{h} \times 10^{-3}$} & \multirow{2}{*}{$\Delta W_{s}$} \\
\hline & $30^{\circ} \mathrm{C}$ & $40^{\circ} \mathrm{C}$ & & & & $30^{\circ} \mathrm{C}$ & $40^{\circ} \mathrm{C}$ & & & & $30^{\circ} \mathrm{C}$ & $40^{\circ} \mathrm{C}$ & & \\
\hline 0.366 & 0.26 & 0.35 & -1.6 & -5.8 & 0.473 & 0.54 & 0.46 & 1.5 & 4.1 & 0.421 & 0.47 & 0.39 & 1.6 & 4.4 \\
\hline 0.430 & 0.11 & 0.12 & -0.21 & -0.92 & 0.567 & 0.52 & 0.46 & 1.1 & 2.8 & 0.675 & 0.38 & 0.72 & -6.4 & -22.4 \\
\hline 0.522 & 0.12 & 0.07 & 0.94 & 2.8 & 0.687 & 0.48 & 0.41 & 1.3 & 3.6 & 0.714 & 0.42 & 0.68 & -5.1 & -18.2 \\
\hline 0.593 & 0.21 & 0.11 & 1.9 & 5.9 & 0.814 & 0.41 & 0.35 & 1.2 & 3.4 & & & & & \\
\hline
\end{tabular}


that the temperature-dependency of the activity of solvent hardly appeared at all in some concentrations.

The relations between the interaction parameter $\chi_{1}$ and the reciprocal of absolute temperature gave straight lines, as shown in Figures 4 and 5. The heat term $\left(\Delta W_{h}\right)$ and the entropy term $\left(\Delta W_{s}\right)$ in $\chi_{1}$ obtained from these graphs are shown in Tables I and II.

Recently, B. E. Eichinger and P. J. Flory have pointed out that a part of the standard state free energy change arises from the contribution of the equation-of-state, which is responsible for almost all of the concentration dependence of $\chi_{1}{ }^{16}$ And it has been found that polysiloxane chains, which are coiled into helices $^{12}$ in the pure state, are easily deformable in the dissolved state. ${ }^{17,18}$ Then, the polysiloxane solution is subject to the contribution from the equation-of-state, characteristic of the kind of solvent.

The values of $\chi_{1}$ in the system of ethyl benzene for polydimethylsiloxane depended markedly on concentration. This system, in which $\Delta W_{h}$ and $\Delta W_{s}$ were found both in large negative values, exhibits a lower critical solution temperature in a wide range of concentrations. ${ }^{19}$ It is expected in such a solution that $\chi_{1}$ contains the large negative contribution from the equation-of-state. ${ }^{20}$

On the other hand, in the systems of $n$-hexane, $n$-octane, benzene and toluene for polydimethyloxane, $\chi_{1}$ was found to be independent of concentration at and near the middle temperature observed, $33^{\circ} \mathrm{C}$. In these systems, absolute values of $\Delta W_{s}$ are small, and the value of $\Delta W_{s}$ varies in proportion to the variation of $\Delta W_{h}$ with concentration. Namely, corresponding $\Delta W_{h}$ and $\Delta W_{s}$ are plotted on an almost straight line in the vicinity of origin, as shown in Figure 6. Then, the variations of $\Delta W_{h}$ and $T \Delta W_{s}$ with concentration may compensate one another.

The same relation in the silicone resin solution is apparently a straight line, as shown in Figure 7, and this result accounts for the concentration-independence of $\chi_{1}$ near $33^{\circ} \mathrm{C}$.

The benzene solution indicates that the negative value of $\Delta W_{h}$ in lower concentrations inverts its sign at higher concentrations. The

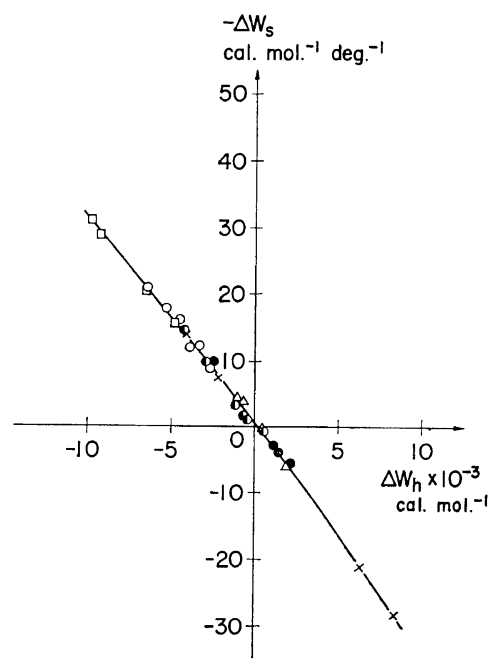

Figure 6. Relation between $\Delta W_{h}$ and $\Delta W_{s}$ for polydimethylsiloxane-solvent systems: $(\bullet) n$-hexane, (O) $n$-heptane, $(\times)$ n-octane, $(\triangle)$ benzene, $(\triangle)$ toluene, and $(\square)$ ethylbenzene.

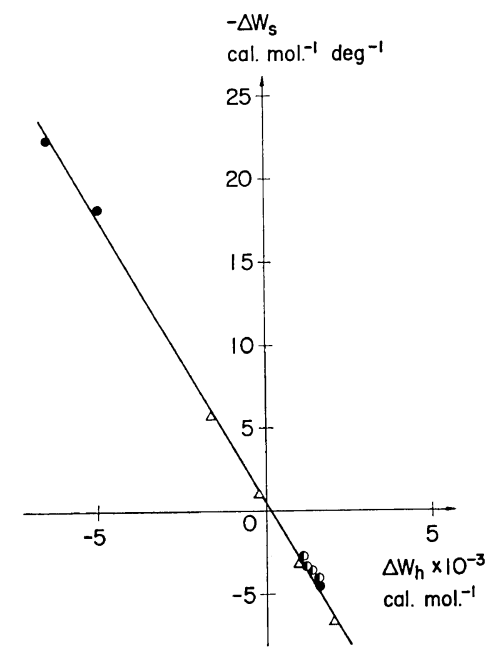

Figure 7. Relation between $\Delta W_{h}$ and $\Delta W_{s}$ for silicone resin-solvent systems: $(-n$-octane, $(\triangle)$ benzene, and (\$) toluene.

same trend has been found in the polyisobutylene solution in cyclohexane ${ }^{21}$ and $n$-pentane. ${ }^{20}$ However, the positive values of $\Delta W_{h}$ at higher concentrations of this solution are too uncertain to enable conclusions to be drawn.

The large positive values of $\Delta W_{h}$, as found in the solution of polydimethylsiloxane in $n$ hexane and in that of silicone resin in $n$-octane, 


\section{A. Muramoto}

predict the limited miscibility that agrees with the observed phase separation in lower concentrations.

The graph, in which $\Delta W_{h}$ are plotted against the corresponding $\Delta W_{s}$, shows a curve for the polydimethylsiloxane solution, whereas it shows a straight line for the silicone resin solution. However, the relations between $\Delta W_{h}$ and $\Delta W_{s}$ may be considered to be a straight line in the range of small $\Delta W_{h}$ and $\Delta W_{s}$, either in the solution of polydimethylsiloxane or in that of silicone resin. The above graphs show readings nearly through the origin. This trend agrees with Takenaka's data. ${ }^{9}$

Takenaka has obtained the above data which were independent of the molecular weight of nitrocellulose. ${ }^{9}$ It is confirmed in this experiment, that the said relations between $\Delta W_{h}$ and $\Delta W_{s}$ are independent of the solvent, although non-polar, and of concentration.

\section{CONCLUSION}

The thermodynamic properties of two types of polysiloxane in non-polar solvent systems were studied. It was found that the values of $\chi_{1}$ and its temperature dependency are characteristic of the combination of polysiloxane and solvent. The relation between the heat term $\Delta W_{h}$ and the entropy term $\Delta W_{s}$ was represented with a smooth curve for each polysiloxane solution. The said relations were confirmed to be independent of the concentration and the kind of solvent, although the said solvents are limited to being non-polar. It was found, furthermore, that the said curve goes nearly through the origin, for each polysiloxane solution.

Acknowledgements. The author wishes to thank Mr. Y. Miura and Mr. S. Miyake of the Material Research Laboratory of Toshiba for permitting him to carry on this research for many years and to publish these results. $\mathrm{He}$ is also much indebted to Professor A. Nakajima of the Department of Polymer Chemistry, Kyoto University, who offered him many helpful suggestions and examined his manuscript in preparing this paper. In addition, he is grateful to Dr. T. Takamura for helpful advice.

\section{REFERENCES}

1. P. J. Flory, "Principles of Polymer Chemistry" Cornell University Press, Ithaca, N. Y., 1953, Chapter 12.

2. G. Gee and L. R. G. Treloar, Trans. Faraday Soc., 38, 147 (1942).

3. S. Prager, E. Bagley, and F. A. Long, J. Amer. Chem. Soc., 75, 2742 (1953).

4. P. J. Flory and H. Daoust, J. Polym. Sci., 25, 429 (1957).

5. G. Gee, J. Chem. Soc., 1947, 280.

6. C. E. H. Bawn, R. F. J. Freeman, and A. R. Kamaliddin, Trans. Faraday Soc., 46, 677 (1950).

7. C. Booth, G. Gee, M. N. Jones, and W. D. Tayler, Polymer, 5, 353 (1964).

8. C. Booth, G. Gee, G. Holden, and G. R. Williamson, Polymer, 5, 343 (1946).

9. H. Takenaka, J. Polym. Sci., 24, 321 (1957).

10. See footnotes of P. J. Flory, "Principle of Polymer Chemistry", Cornell University Press, Ithaca, 1953, p 510.

11. E. A. Guggenheim, Trans. Faraday Soc., 44, 1007 (1948).

12. H. W. Fox, P. W. Taylor, and W. A. Zisman, Ind. Eng. Chem., 39, 1401 (1947); E. C. Warrick, M. J. Hunter, and M. J. Barry, ibid., 44, 2196 (1952); S. M. Ohlberg, L. E. Alexander, and E. L. Warrick, J. Polym. Sci., 27, 1 (1958); E. G. Rochow, "An Introduction to the Chemistry of the Silicones"' John Wiley and Sons, Inc., New York, N.Y., 1947, Chapter 4, p 60.

13. J. Barry, J. Appl. Phys., 17, 1020 (1946).

14. Deutshen Chemischen Gesellschaft, "Beilsteins Hundbuch der Organischen Chemie", Band 1 (1928) u. Band 5 (1930), Erstes Ergänzungswerk, Julius Springer, Berlin.

15. M. L. Huggins, Ind. Eng. Chem., 35, 216 (1943).

16. B. E. Eichinger and P. J. Flory, Trans. Faraday Soc., 64, 2035 (1968).

17. P. J. Flory, L. Mandelkern, J. B. Kinsinger, and W. B. Schultz, J. Amer. Chem. Soc., 74, 3364 (1952).

18. M. J. Newing, Trans. Faraday Soc., 46, 613 (1950).

19. C. H. Baker, W. B. Brown, G. Gee, J. S. Rowlinson, D. Stubley, and R. E. Yeadon, Polymer, 3, 213 (1962).

20. B. E. Eichinger and P. J. Flory, Trans. Farady Soc., 64, 2066 (1968).

21. B. E. Eichinger and P. J. Flory, ibid., 64, 2061 (1968). 